\title{
Oncology Research and Treatment
}

\section{Meetings and Conferences}

Oncol Res Treat 2015;38:549-550

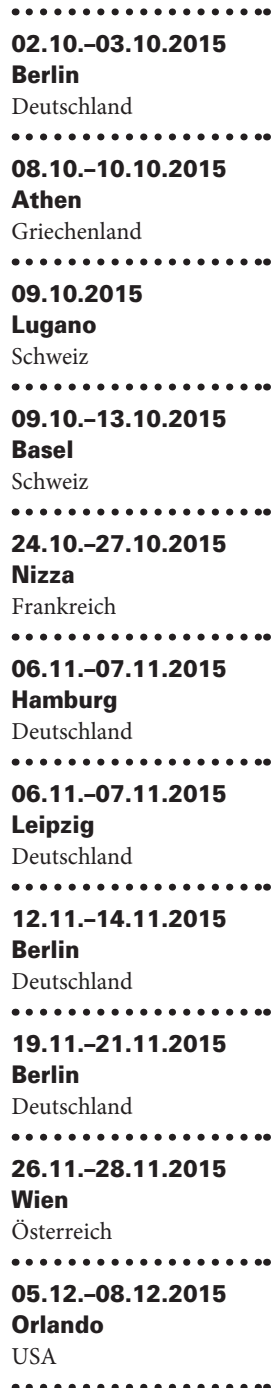

08.12.-12.12.2015

San Antonio

USA

19.12.2015

München

Deutschland

•.................

11.02.-13.02.2016

Düsseldorf

Deutschland

..................

18.02.-20.02.2016

St. Gallen

Schweiz

.................

24.02.-27.02.2016

Berlin

Deutschland

\section{DGHT-Symposium: Hyperthermie - Perspektiven und Grenzen}

20th World Congress on Advances in Oncology and 18th International Symposium of Molecular Medicine

VIII Edition Senoforum

Jahrestagung der Deutschen, Österreichischen und Schweizerischen Gesellschaften für Hämatologie und Medizinische Onkologie

ESGO 19th International Meeting - European Gynaecological Oncology Congress 2015

23. Endo Club Nord

UroCurriculum: Prostatakarzinom II

Fortbildungszertifikat der Deutschen Akademie für Senologie -

Theoriemodule

12. AIO-Herbstkongress - Update Medical Oncology

18. Zentraleuropäisches Seminar: Methodik klinischer Prüfung in der Onkologie

57th ASH Annual Meeting and Exposition

38th CTRC-AACR Annual San Antonio Breast Cancer Symposium (SABCS 2015)

IX. Wissenschaftliches Wintersymposium

Aktuelle Entwicklungen bei Gynäkologischen Tumoren und Brustkrebs

18. Internationales Endoskopie Symposium Düsseldorf

26. Ärzte-Fortbildungskurs in Klinischer Onkologie

32. Deutscher Krebskongress 2016
Auskunft: www.hyperthermie-kongress.de

\section{Auskunft:}

http://www.spandidos-publications.com/pages/conference

Auskunft: www.senoforum.ch/

Auskunft: www.haematologie-onkologie-2015.com

Auskunft: www.esgo2015.esgo.org

Auskunft: www.endoclubnord.de

Auskunft:www.uro-akademie.de

Auskunft: www.akademie-senologie.de

Auskunft: www.aio-herbstkongress.de

Auskunft: www.acr-itr-vienna.at

Auskunft: www.hematology.org/Meetings

Auskunft:www.sabcs.org

Auskunft:www.wintersymposium-muenchen.de

Auskunft: www.endo-duesseldorf.com

\section{Auskunft:}

www.oncoconferences.ch/dynasite.cfm?dsmid=113358

Auskunft: www.dkk2016.de

\section{KARGER}

Fax +497614520714

Information@Karger.com

www.karger.com 
29.02.-02.03.2016

Aachen

Deutschland

•........

08.04.-10.04.2016

New Delhi

Indien

... . . . . . . . .

Münster

Deutschland

21.04.-23.04.2016

Warth b. Frauenfeld

Schweiz

..................

28.04.-30.04.2016

Dresden

Deutschland

...............

25.06.2016

Wiesbaden

Deutschland

00.0000000 .0 .0$.

01.09.-02.09.2016

St. Gallen

Schweiz

...............

14.10.-18.10.2016

Leipzig

Deutschland

.................

04.11.-05.11.2016

Hamburg

Deutschland

[...............

15.03.-18.03.2017

Wien

Österreich

...................

04.05.-06.05.2017

Mannheim

Deutschland
25. Rehabilitationswissenschaftliches Kolloquium in Aachen. Deutscher Kongress für Rehabilitationsforschung. Thema „Gesundheitssystem im Wandel - Perspektiven der Rehabilitation"

12th International Conference of Asian Clinical Oncology (ACOS)

62. Kongress der Nordrhein-Westfälischen Gesellschaft für Urologie e.V.

20. Internationales Seminar: Palliativbetreuung von Tumorkranken

UroAktuell 2016

GI-Oncology 2016 - 12. Interdisziplinäres Update

19. Internationales Seminar: Onkologische Pflege - Fortgeschrittene

Praxis

Jahrestagung der Deutschen, Österreichischen und Schweizerischen Gesellschaften für Hämatologie und Medizinische Onkologie

24. Endo Club Nord

Kongress für Life-Endoskopie

15th St Gallen International Breast Cancer Conference - Primary Therapy of Early Breast Cancer Evidence, Controversies, Consensus

UroAktuell 2017
Auskunft: www.reha-kolloquium.de

Auskunft:www.acos2016.com

Auskunft: www.nrwgu-kongress.de

\section{Auskunft:}

www.oncoconferences.ch/dynasite.cfm?dsmid=113384

Auskunft: www.uro-akademie.de

Auskunft:www.gi-oncology.de

\section{Auskunft:}

www.oncoconferences.ch/dynasite.cfm?dsmid=113309

Auskunft: www.haematologie-onkologie-2016.com

Auskunft: www.endoclubnord.de

Auskunft: www.oncoconferences.ch/

Auskunft: www.uro-akademie.de 